\title{
ASSOCIATION AFFAIRS
}

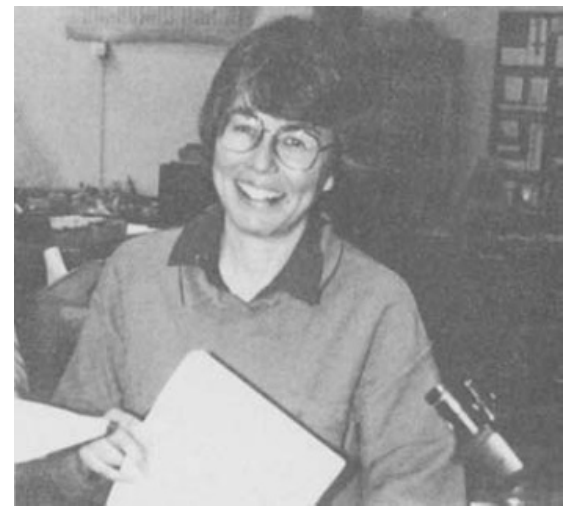

Elisabeth Anne Wheeler

\section{New IAWA Bulletin editor}

Dr. Elisabeth A. Wheeler from the School of Forestry, North Carolina State University, Raleigh, U.S.A., has been appointed an editor of the IAWA Bulletin by the IAWA Council. She will process about $50 \%$ of all manuscripts through the refereeing and other editorial stages. Her work will not only substantially reduce the workload in Leiden, but also add new expertise beneficial to improve the standards of our journal. For practical reasons contributors are requested to continue to submit all manuscripts to the Leiden office. We look forward to fruitful cooperation.

Pieter Baas

\section{New Members}

Mr. Jou-Horng Chern

National Taiwan University

Department of Forestry

Taipei 107

Taiwan

Dr. Igor Cunderlík

University of Forestry \& Wood Technology

96053 Zvolen

Czechoslovakia

Mr. Arend C. Diesel

Vuldersstraat 39

8000 Brugge

Belgium

Mr. James J. Farrar

University of Wisconsin

Department of Botany

132 Birge Hall

Madison, WI 53706

U. S. A.

Prof. Dr. C.S. Han

Chonbuk National University

Dept of Forest Products \& Technology

Chonju Chonbuk 560-756

Korea (South)

Prof. Friedemann Hellwig

Fachhochschule Köln

FB Restaurierung \& Konservierung

Claudiusstrasse 1

5000 Köln 1

Germany
Dr. Yasuhiko Hirakawa

Forestry \& Forest Products Research

Institute

P.O. Box 16

Tsukuba Norin, Ibaraki 305

Japan

Mr. P. Immerzeel

IJsselstraat 34

$4335 \mathrm{KC}$ Middelburg

The Netherlands

Ms. Ilona Koloszar-Peszlen

Virginia Tech

825 Orchard St., R-7 Draper's Meadow

Blacksburg, VA 24060

U. S. A.

Prof. V.I. Onegin

Leningrad Forest Technical Academy

Institutsky per. 5

Leningrad 194018

U. S. S. R.

Mr. Stan Schmidt

Luthier

112 Evergreen

Elmhurst, IL 60126

U. S. A.

Dr. Uwe Schmitt

Institut für Holzbiologie und Holzschutz

Leuschnerstrasse 91

2050 Hamburg 80

Germany 
Ms. Gudrun Weiner

c/o Universităt Hamburg

Ordinariat für Holzbiologie

Leuschnerstrasse 91

2050 Hamburg 80

Germany

Dr. Rupert Wimmer

Universităt für Bodenkultur

Zentrum für Umwelt- und Naturschutz

Gregor-Mendel-Strasse 33

1180 Vienna

Austria

Dr. Daniele Zanzi

Fitoconsult

Via Orazio 5

21100 Varese

Italy

\section{Changes of address}

Dr. Hiran S. Amarasekera

University of Sri Jawawardenepura

Department of Botany

Forest Resources Development Project

Nugegoda

Sri Lanka

Ms. V. Angyalossy Alfonso

Departamento de Botânico

Instituto de Biociências

Universidade de São Paulo

Caixa Postal 11.461, CEP 05499

01000 São Paulo

Brazil

Dr. V. Dubbel

Hessische Forstliche Versuchsanstalt

Prof. Oelkers Strasse 6

3510 Hann. Münden

Germany

Mr. Wolfgang Gard

St. Laurensbaai 38

2904 AC Capelle a/d IJssel

The Netherlands

Ing. P.B. Laming

TNO Centre for Paper \& Board Research

P.O. Box 6034

$2600 \mathrm{JA}$ Delft

The Netherlands
Dr. H.-H. Leuschner

Schillerstrasse 31

3400 Gottingen

Germany

Miss A.E. Luchi

Instituto de Botânica

Caixa Postal 4005

01051 Såo Paulo - SP

Brazil

Ms. Yvonne Möller-Lindenhof

Institut für Holzbiologie und Holzschutz

Leuschnerstrasse 91

2050 Hamburg 80

Germany

Dr. Wonkyo Park

Chungbuk National University

Department of Forest Products

College of Agriculture

Cheungju 360-763

Korea (South)

Dr. David C. Ritter

732 Illinois

Geneva, IL 60134

U. S. A.

Ms. Eva Sorfa

Forestek

P.O. Box 395

Pretoria 0001

South Africa

Mr. Francisco J. de Vasconcellos

INPA/CPPF

Caixa Postal 478

69011 Manaus - Amazonas

Brazil

Mr. Yi-Chung Wang

National Taiwan University

Department of Forestry

Taipei 107

Taiwan

Mr. A.P. Wilkins

44 Cave Road

South Strathfield

Sydney - 2136

Australia 